\title{
Translation factor control of ribosome conformation during start codon selection
}

\author{
Katsura Asano ${ }^{1,5}$ and Matthew S. Sachs $s^{2,3,4}$ \\ ${ }^{1}$ Molecular Cellular and Developmental Biology Program, Division of Biology, Kansas State University, Manhattan, Kansas \\ 66506, USA; ${ }^{2}$ Department of Environmental and Biomolecular Systems, Oregon Health and Science University, Beaverton, \\ Oregon 97006, USA; ${ }^{3}$ Department of Molecular Microbiology and Immunology, Oregon Health and Science University, \\ Portland, Oregon 97201, USA
}

Eukaryotic translation initiation factors (eIFs) function at multiple steps. They enable the small $40 \mathrm{~S}$ ribosome subunit to bind to initiator tRNA and mRNA, and scan to and select an initiation codon on the mRNA. They facilitate joining of the large 60S ribosome subunit, at which point the initiation phase of translation ends with the initiator tRNA in the $\mathrm{P}$ (peptidyl) site, and the ribosome poised to accept a tRNA into its A (aminoacyl) site (Kapp and Lorsch 2004; Pestova et al. 2007). There are at least 10 eIFs, and many of them (eIF1, eIF1A, eIF2, eIF3, eIF4F, eIF5, and eIF5B) are direct components of the ribosomal preinitiation complex (PIC). eIF2 and eIF3 are well-characterized multimeric factors that bind directly to Met-tRNA ${ }_{i}{ }^{\text {Met }}$ and the $40 \mathrm{~S}$ subunit, respectively.

While considered relatively large, the 40S subunit has only a limited functional space to bind translation factors. eIF1 and eIF1A are small single-polypeptide factors that bind directly to the ribosome at or near the decoding site. They are thought to directly regulate ribosome conformation. An elegant combination of genetic and biochemical studies reported in the previous issue of Genes \& Development from the Hinnebusch, Lorsch, and Pestova laboratories (Cheung et al. 2007) provides substantial evidence that these factors indeed regulate the ribosome conformational rearrangement in response to start codon selection. This report and other recent reports on the structures and functions of yeast initiation factors lead to a deeper understanding of how eIFs bind the ribosome productively, communicate with each other to enable initiation, and regulate the ribosome's conformation and activity to maintain initiation fidelity.

The salient points concerning translation initiation via scanning (outlined in Fig. 1) that are important for considering the functions of eIFs in start site selection can be summarized as follows. The ternary complex (TC) is composed of eIF2, GTP, and Met-tRNA ${ }_{i}^{\text {Met }}$. It associ-

Correspondence.

${ }^{4}$ E-MAIL msachs@ebs.ogi.edu; FAX (503) 690-1464.

${ }^{5}$ E-MAIL kasano@ksu.edu; FAX (785) 532-6653.

Article is online at http://www.genesdev.org/cgi/doi/10.1101/gad.1562707. ates with the $40 \mathrm{~S}$ ribosome subunit with the assistance of eIF1, eIF1A, and eIF3. TC, eIF1, eIF3, and eIF5 together can be isolated as a multifactor complex (MFC) in yeast (Asano et al. 2000); a similar complex may be present in mammals (LeFebvre et al. 2006). There are at least two possible pathways for $43 \mathrm{~S}$ assembly as depicted in Figure 1 , one of which involves preformed MFC. The 43S PIC that is formed by these interactions is composed of the $40 \mathrm{~S}$ subunit, MFC, and eIF1A. The $43 \mathrm{~S}$ PIC is recruited to the mRNA by eIF4F, which is associated with the mRNA $\mathrm{m}^{7} \mathrm{G}$-cap and poly $(\mathrm{A})$ tail through association with poly(A)-binding protein; the PIC in association with mRNA becomes the 48S PIC [eIF4F, poly(A), and PABP are not shown in Fig. 1]. eIF3 also is important for PIC binding to mRNA (Kolupaeva et al. 2005; Siridechadilok et al. 2005; Hinnebusch 2006; Jivotovskaya et al. 2006). The PIC scans the mRNA; upon determining it has reached a start codon, it releases the $\mathrm{P}_{\mathrm{i}}$ formed by hydrolysis of GTP in the TC, and also releases eIF2.GDP, leaving Met-tRNA ${ }_{i}{ }^{\text {Met }}$ positioned at the start codon. As discussed below, eIF1 also dissociates from the ribosome during selection of the start codon. A second GTP-binding factor, eIF5B, then facilitates 60S subunit joining. The guanine nucleotide exchange factor eIF2B recycles eIF2 $\bullet \mathrm{GDP}$ to eIF2•GTP, because only the latter binds Met-tRNA ${ }^{\text {Met }}$.

\section{An overview of yeast genetic studies on eIF functions}

Much of our understanding concerning mechanisms of eukaryotic translation initiation originates from genetic analyses using Saccharomyces cerevisiae. The studies in the previous issue rely partly on two classic phenotypes (discussed further in Donahue 2000; Hinnebusch 2005) used to select for translation initiation defects in this organism: $\mathrm{Sui}^{-}$(suppressors of initiator codon) and $\mathrm{Gcd}^{-}$ (general control derepressed). The isolation of genes in which defects caused the Sui ${ }^{-}$phenotype was originally accomplished by Thomas Donahue and colleagues (Donahue et al. 1988). The purpose of those experiments was to identify factors involved in translation start site 


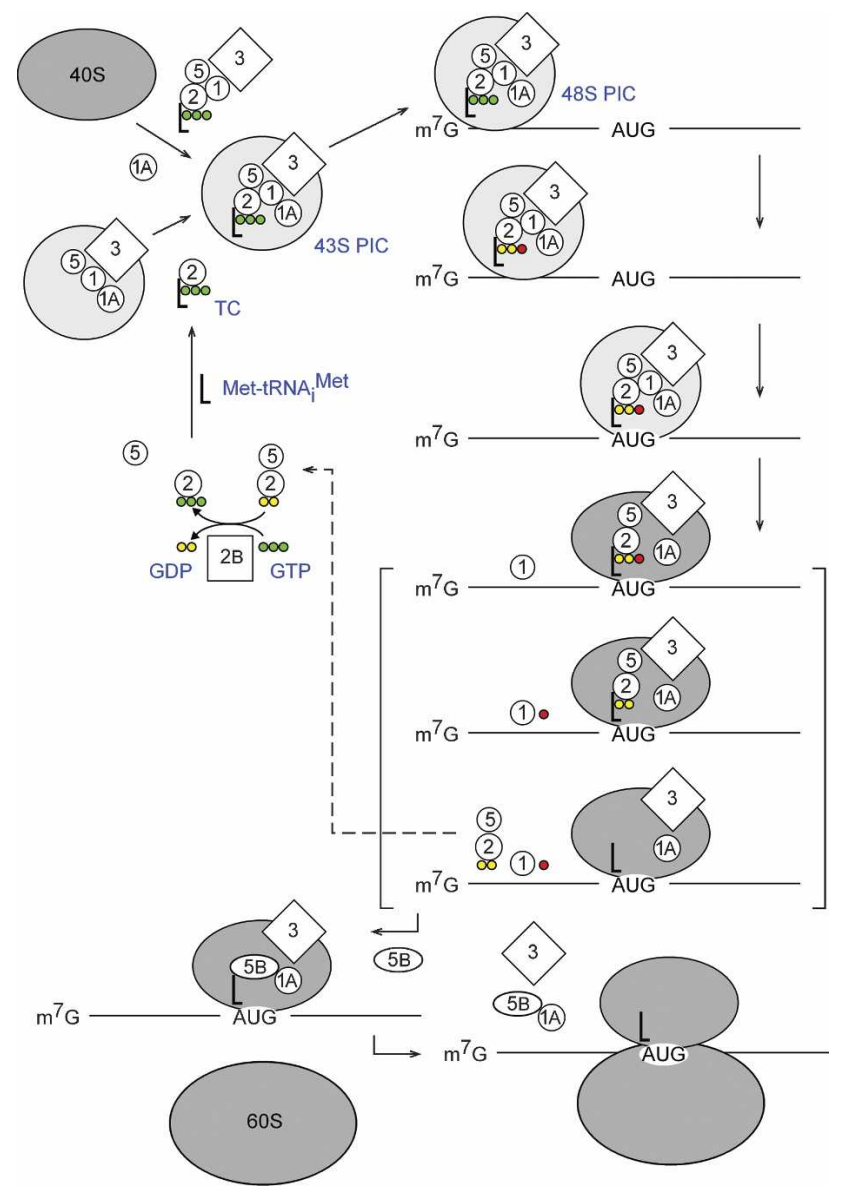

Figure 1. Simplified model for translation initiation. 43S PIC formation by two different pathways is indicated. The 43S PIC is loaded at the mRNA 5' end with the assistance of eIF4F (not shown) to create the $48 \mathrm{~S}$ PIC. eIF2 in the $48 \mathrm{~S}$ PIC is bound to GTP (indicated with three green circles), GTP in equilibrium

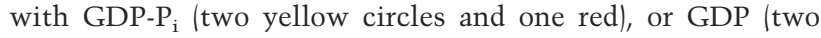
yellow circles). Factors, GDP, and $\mathrm{P}_{\mathrm{i}}$ (red circle) that leave the ribosome are indicated to the left of the ribosome. The bracketed intermediates represent the changes that occur when the PIC converts from an open to a closed conformation; the conformational change is indicated by alterations to the 40S subunit's shape and shading. The exchange of GDP bound to eIF2 for GTP, with release of eIF5, by eIF2B is also indicated, as is the reformation of TC by binding of eIF2 $\bullet$ GTP to Met-tRNA ${ }_{i}{ }^{\text {Met }}$.

selection, which at that time had been established in yeast to occur at the most 5'-proximal AUG in the mRNA (Stewart et al. 1971) and appeared to be governed by the scanning model proposed by Marilyn Kozak (Kozak 1978). The yeast strain used contained his4- and his4-1acZ alleles in which the authentic AUG start codon was changed to AUU (Donahue and Cigan 1988). Potential suppressors were then genetically identified by selecting for growth of cells on medium lacking histidine and screening for the ability of such cells to produce LacZ by color assay using indicator plates containing $\mathrm{X}-\mathrm{Gal}$. The first of such analyses revealed that three loci were affected: sui1 and sui2 were recessive, and SUI3 mutations were dominant (Castilho-Valavicius et al.
1990). These genes specify eIF1 and the $\alpha$ and $\beta$ subunits of eIF2, respectively; the Sui ${ }^{-}$mutations isolated in each increased initiation at a UUG codon that is used when the authentic AUG is eliminated by mutation to AUU (Donahue 2000 and references therein). Two additional genes in which mutations caused the Sui ${ }^{-}$phenotype were subsequently identified: SUI4 (alleles of which are $\mathrm{Gcd}^{-}$) specifies eIF2 $\gamma$, and SUI5 specifies eIF5. The his4303 (AUU) allele used for the original isolations is also used by Cheung et al. (2007) to test whether eIF1 mutants allowed sufficient initiation to enable growth in media lacking histidine; however, his4-luc (luciferase) reporters have been used in addition to his4-lac $Z$ reporters for quantitative analysis of how these mutants affected initiation at UUG relative to AUG.

The general control derepressed $\left(\mathrm{Gcd}^{-}\right)$phenotype reflects increased expression of GCN4, a transcriptional activator of many amino acid biosynthetic genes. This phenotype can result from mutations affecting translation initiation because GCN4 expression is controlled by upstream ORFs (uORFs) that govern translation initiation at the GCN4 start codon. In this control mechanism, ribosomes initiate translation efficiently at the uORF1 start codon, and then following termination of uORF1 translation, can reinitiate downstream either at another uORF start codon or at the GCN4 start codon. The $\mathrm{Gcd}^{-}$phenotype enables cells to grow in the presence of toxic amino acid analogs that can be incorporated in place of amino acids in polypeptides; derepression of GCN4 enables increased production of amino acids to successfully compete with the analog for incorporation into polypeptides (for review, see Hinnebusch 2005). A $\mathrm{Gcn}^{-}$(general control nonderepressible) phenotype arises when GCN4 expression cannot be derepressed (e.g., by mutation in GCN4 itself; hence its name). The normal derepression response of GCN4 requires GCN2, which specifies a kinase that phosphorylates the $\alpha$ subunit of eIF2 and responds to increased levels of deacylated tRNA (and thus amino acid limitation). This phosphorylation event changes eIF2 from acting as a substrate of eIF2B to acting as a competitive inhibitor of eIF2B, and thereby decreases formation of TC. This leads to decreased reinitiation at uORF4 and increased reinitiation at GCN4 instead. gcn $2 \Delta$ mutants are thus $\mathrm{Gcn}^{-}$. Of particular interest here are mutations that affect translation initiation that enable a $\mathrm{Gcd}^{-}$phenotype in a gcn2s background; some of these mutations should affect initiation even when normal levels of functional TC are present, for example, by slowing TC binding to the $40 \mathrm{~S}$ subunit.

\section{eIF1 as a regulator of start site selection}

eIF1 is a small protein (12 kDa) that participates in multiple aspects of initiation. It is a component of the MFC and stimulates the recruitment of TC to the 40S subunit (Singh et al. 2004). Some of the mutations in eIF1 characterized in the present study (Cheung et al. 2007) destabilize the MFC as well. Importantly, eIF1 also plays pivotal roles in regulating the PIC in response to start codon selection. First, during the process of start codon 
selection, the eIF2-mediated hydrolysis of GTP in the TC that must occur is assisted by eIF5, which acts as a GTPase activator protein (GAP) (Das et al. 2001; Paulin et al. 2001; Majumdar and Maitra 2005), and eIF1 appears to negatively affect this interaction (see below). But surprisingly, start codon selection is not strictly coupled to the hydrolysis event; it appears that hydrolysis can occur in the PIC during scanning, but the $\mathrm{P}_{\mathrm{i}}$ formed is not released and instead remains associated, so that there is equilibrium between GTP and GDP-Pi associated with TC (Fig. 1). Start codon selection is more tightly coupled to the rate of $\mathrm{P}_{\mathrm{i}}$ release than to the hydrolysis event (Algire et al. 2005). That is, even when GTP is hydrolyzed to GDP in the 48S PIC prior to start codon recognition, the $\mathrm{P}_{\mathrm{i}}$ does not dissociate until start codon recognition occurs, and it is $P_{i}$ release that is most important for start codon recognition.

How does eIF2 respond to start codon selection and release $\mathrm{P}_{\mathrm{i}}$ ? The model supported by the work described in the previous issue by Cheung et al. (2007) is that there is a conformational change in the PIC, or perhaps the ribosome itself, upon recognition of AUG (or an alternative start codon), from an "open" to a "closed" conformation, associated with the release of eIF1, which ultimately leads to $P_{i}$ release. The open conformation of the PIC is the scanning-competent conformation that allows efficient sliding of mRNA along the mRNA-binding path of the 40S subunit. Many of the eIFs that bind to the ribosome are thought to regulate the open conformation either positively or negatively. The closed conformation of the PIC does not scan; instead, it tightly binds the selected start codon. Start codon recognition by the PIC via base-pairing to the $\mathrm{RNA}_{\mathrm{i}}{ }^{\mathrm{Met}}$ anti-codon is thought to promote this conformational change. Associated with the change to a closed conformation is the release of eIF1, $\mathrm{P}_{\mathrm{i}}$, eIF2•GDP, and eIF5 (the latter possibly complexed together) (Singh et al. 2006), and the capacity to bind eIF5B in a way that catalyzes 60 S subunit joining.

This model enables an explanation for why Sui ${ }^{-}$mutations in eIF5 or the eIF2 $\gamma$ subunit, each of which increases the rate of GTP hydrolysis in the TC, relax the stringency of start codon selection. By forcing the equilibrium between eIF2 $\bullet$ GDP-Pi and eIF2 $\bullet$ GTP associated with the scanning ribosome toward the eIF2 $\bullet \mathrm{GDP}-\mathrm{Pi}$ form, these mutations increase the likelihood that the PIC erroneously responds to suboptimal base-pairing with UUG with $P_{i}$ release.

The two-conformation hypothesis for the scanning ribosome was first proposed by Tatyana Pestova and colleagues (Pestova et al. 1998; Pestova and Kolupaeva 2002; Lomakin et al. 2003) as the result of their analyses of in vitro reconstituted PIC using mammalian components in which eIF1 and eIF1A were shown to promote scanning, and eIF1 to be important in blocking selection of non-AUG start codons. The first biophysical evidence for a conformational change in the PIC was obtained by FRET analyses of labeled eIF1A and eIF1 (Maag et al. 2005 ) and very recently by cryo-EM analysis of the 40S/ eIF1/eIF1A complex displaying an open conformation compared with a closed conformation of unbound $40 \mathrm{~S}$ subunit (Passmore et al. 2007). Importantly, in the former FRET study (Maag et al. 2005), it was observed that the conformational change resulted in the release of eIF1 from the ribosome. Thus, start codon recognition is accompanied by both $\mathrm{P}_{\mathrm{i}}$ and eIF1 release. For example, eIF1 containing the mutation $G 107 R$ slowly dissociates the PIC in vitro, and $\mathrm{P}_{\mathrm{i}}$ release measured with the PIC containing this form of eIF1 is correspondingly slow (Algire et al. 2005). These and other kinetic analyses accomplished by the Lorsch laboratory indicate that eIF1 release controls $\mathrm{P}_{\mathrm{i}}$ release and not vice versa (Algire et al. 2005). As eIF1 binds the ribosome at a site close to but distinct from the P site (Lomakin et al. 2003), it is presumed that the local conformational change of the ribosome induced by codon-anti-codon pairing directly results in eIF1 release, and this triggers the cascade of release of $\mathrm{P}_{\mathrm{i}}$, eIF2•GDP, and eIF5, forming the closed PIC conformation positioned at the start codon.

Previous genetic studies from the Hinnebusch laboratory (Valásek et al. 2004) indicated that eIF1 overexpression suppresses the Sui ${ }^{-}$phenotype of the SUI5 (eIF5)G31R mutant that was known to increase the GTP hydrolysis for eIF2 in a simplified PIC in vitro (Huang et al. 1997). This is consistent with the model that the direct interaction of eIF1 with the PIC (likely interaction with the $40 \mathrm{~S}$ ribosome subunit) constitutes a second important part of PIC regulation. Biochemical data support the idea that eIF1 inhibits the GAP function of eIF5 at nonAUG codons (Unbehaun et al. 2004; Algire et al. 2005). Suppression is interpreted to arise as a consequence of increased binding of eIF1 to the PIC by a mass-action argument: Increased binding (1) counteracts the activated GAP function arising from the eIF5 G31R mutation, since eIF1 inhibits eIF5 GAP activity, and/or (2) reduces the likelihood of PICs committing to the closed conformation through the loss of eIF1.

\section{Evidence for eIF1 release as a critical checkpoint of start codon selection}

In the present study, Cheung et al. (2007) directly tested the hypothesis that eIF1 release is a key step in start codon selection in vivo. First, the powerful tool of the in vitro reconstitution system developed by the Lorsch laboratory was used to assay the effect of mutations strategically placed in different domains of eIF1. These results were then carefully compared with the effects of the same eIF1 mutations observed in vivo. The in vitro system uses eIF1, eIF1A, TC (recall that TC is comprised of eIF2, GTP and Met-tRNA ${ }_{i}{ }^{\text {Met }}$, a model mRNA containing either AUG or UUG to use as a start codon, and the $40 \mathrm{~S}$ subunit. We refer to the in vitro complex formed with these components as $48 \mathrm{~S}(\mathrm{AUG})^{\star}$ or $48 \mathrm{~S}(\mathrm{UUG})^{*}$, respectively, and the complex lacking the mRNA as $43 S^{\star}$. This system can measure $(1)^{35}$ S-Met-labeled TC loading to the 40S subunit (by gel retardation assay; the label is in the aminoacylated tRNA); (2) ribosome conformational change (by a rapid phase of FRET loss between eIF1 and eIF1A, each of which has been fluorescently tagged); (3) eIF1 release (by a slow phase of FRET loss 
between eIF1 and eIF1A); (4) $\left[\gamma_{-}{ }^{32} \mathrm{P}\right] \mathrm{GTP}$ hydrolysis per se (by a fast phase of ${ }^{32} \mathrm{P}_{\mathrm{i}}$ release from ${ }^{32} \mathrm{P}-43 \mathrm{~S}^{\star}$ mixed with saturating eIF5 and mRNA using a rapid quench device); and $(5)^{32} \mathrm{P}_{\mathrm{i}}$ release from ${ }^{32} \mathrm{P}-48 \mathrm{~S}^{\star} \mathrm{PIC}$ [by a slow phase of ${ }^{32} \mathrm{P}_{\mathrm{i}}$ release from ${ }^{32} \mathrm{P}-43 \mathrm{~S}^{\star}$ treated as for $\left.(4)\right]$.

One caveat concerning the interpretation of the in vitro data is that the system used lacks factors that are important for initiation in vivo. The reconstituted system used does not include eIF3, so eIF3-dependent processes that would be important in vivo cannot be assayed. The system also lacks eIF4F, which has a critical role in loading the $43 \mathrm{~S}$ complex onto mRNA in vivo, and may have additional roles in regulating TC GTPase activity (He et al. 2003; Majumdar and Maitra 2005).

eIF1 is composed of a 23-residue unstructured $\mathrm{N}$-terminal tail (NTT) and a tightly packed globular domain characterized by two prominent basic surfaces and adjacent hydrophobic surfaces (Fletcher et al. 1999). Cheung et al. (2007) isolated-and examined with comprehensive assays-two well-behaved mutations of eIF1, 9,12 and 93-97, that affected these regions. 9,12 alters the NTT and showed a strong $\mathrm{Gcd}^{-}$phenotype (see below), while 93-97 alters a hydrophobic surface of the globular domain and displayed a weak $\mathrm{Gcd}^{-}$phenotype and a strong Sui ${ }^{-}$phenotype. The 93-97 mutation resulted in increased release of both eIF1 and $P_{i}$ from 48S(AUG)*, consistent with its Sui ${ }^{-}$phenotype. In an independent toeprint assay to analyze start codon recognition, which uses highly purified mammalian translation components, the 93-97 and other Sui- forms of eIF1 produced by the sui1 alleles originally identified by the Donahue group (Yoon and Donahue 1992) were used by Cheung et al. (2007) to replace mammalian eIF1. These Sui- ${ }^{-}$forms of yeast eIF1 in association with mammalian factors increased the toeprint at a GUG codon (wild-type yeast eIF1 did not). Moreover, Cheung et al. (2007) showed that the Sui- ${ }^{-}$phenotype of 93-97 was partially suppressed by overexpression of the altered protein, as were the Sui ${ }^{-}$ phenotypes of the same Donahue sui1 alleles. All of the Sui- mutations reduced the affinity of eIF1 for the PIC in vitro. These data suggest that the deficiencies of the mutant eIF1 binding to the ribosome that results in the Sui ${ }^{-}$ phenotype are overcome by mass action (e.g., by greater eIF1 availability). They also found that 93-97 increased the rate of eIF1 and $P_{i}$ release regardless of whether AUG or UGG was the start codon. This last finding suggests that eIF1 release regulates a step after start codon selection, and is consistent with the idea that eIF1 release is the first response of a ribosome becoming committed to initiate translation at a selected start codon, and that eIF1 release regulates subsequent $\mathrm{P}_{\mathrm{i}}$ release.

Importantly, Cheung et al. (2007) also analyzed an eIF1A-NTT (17-21) mutation that conferred a "hyperaccuracy" phenotype. That is, this eIF1A mutation suppressed the dominant Sui ${ }^{-}$phenotypes of eIF5 and eIF2 $\beta$ mutations (Fekete et al. 2007). Cheung et al. (2007) predicted that this mutation in eIF1A leading to hyperaccuracy would decrease, rather than increase, the rate of eIF1 release; this was observed by using the FRET assay to examine $48 \mathrm{~S}(\mathrm{AUG})^{\star}$ in vitro. Thus, mutations that decreased (eIF1A) or increased (eIF1) the rates of eIF1 release, as observed in vitro, conveyed negative and positive effects, respectively, on non-AUG selection, as observed in vivo, indicative of direct regulation of start codon selection by the release of eIF1.

The weak $\mathrm{Gcd}^{-}$phenotype of 93-97 was suppressed by overexpression of TC by using a high-copy plasmid specifying the three eIF2 subunits and tRNA ${ }_{i}{ }^{\text {Met }}$. The 93-97 mutation reduces the amount of MFC components associated with eIF1 in vivo, as evaluated by analyses of copurifying eIFs following pull-down of His-tagged eIF1. It reduces the amount of MFC components associated with $40 \mathrm{~S}$ subunits in vivo, as evaluated by analyses of factors that remain associated with the $40 \mathrm{~S}$ ribosome subunit following cross-linking with formaldehyde and velocity sedimentation through sucrose gradients. These findings indicate that the hydrophobic surface of eIF1 is important for mediating MFC assembly at least in part by its capacity to bind to eIF3c-NTT. TC overexpression may help abrogate the reduced affinity of initiation complexes containing eIF1(93-97) for TC.

In complementary studies focusing on the structural biology of eIF1 interactions with other components of the PIC, the Wagner and Asano laboratories (M. Reibarkh, Y. Yamamoto, C.R. Singh, F. del Rio, B. Lee, R.E. Luna, M. Ii, G. Wagner, and K. Asano, in prep.) used NMR-mapping studies to show that a continuous basic and hydrophobic surface of eIF1 binds to the eIF5 Cterminal domain (CTD). The eIF1-M4 mutation, which alters the basic residues K100, K101, K104, and H106, which are part of this surface, is lethal; it reduces eIF1 binding to MFC and to $40 \mathrm{~S}$ subunits in vivo. Furthermore, overexpression of the mutant protein in the presence of wild-type eIF1 confers a weak Sui ${ }^{-}$phenotype. These results provide evidence that this surface of eIF1 provides a critical link of eIF1 to the PIC via the interaction with eIF5-CTD. The 93-97 mutation examined by Cheung et al. (2007) alters the hydrophobic area adjacent to the basic surface affected by eIF1-M4. Each of these surfaces is distinct from the ribosome-binding face of eIF1 that was previously defined (Lomakin et al. 2003). Together, these studies define two important functional surfaces of eIF1, one of which binds the ribosome (Lomakin et al. 2003) and the other, eIF5 (Cheung et al. 2007; M. Reibarkh, Y. Yamamoto, C.R. Singh, F. del Rio, B. Lee, R.E. Luna, M. Ii, G. Wagner, and K. Asano, in prep.).

\section{The eIF1-NTT promotes MFC assembly and regulates PIC function}

Elements of the data presented by Cheung et al. (2007) that at face value are difficult to reconcile are the results obtained with mutation of eIF1-NTT residues F9 and F12 (referred to as the 9,12 mutation). Differences between the in vivo and in vitro findings with this mutant contrast with the striking correlation of genetic and biochemical data concerning the behavior of 93-97 and other previously characterized Sui- mutations. The eIF1 $(9,12)$ change causes a dominant $\mathrm{Gcd}^{-}$phenotype. The overexpression of TC by using a high-copy plasmid 
specifying the three eIF2 subunits and tRNA ${ }_{i}{ }^{\text {Met }}$ suppresses the $\mathrm{Gcd}^{-}$phenotype, indicating that 9,12 is interfering with TC recruitment. Consistent with this, in vitro analysis of TC recruitment indicated that, while association of eIF1 $(9,12)$ with the 40 S subunit appeared similar to that of the wild-type form, the 9,12 form significantly reduced the rate of TC binding to $40 \mathrm{~S}$ subunits. But unexpectedly, yeast with this mutation showed no defect in 48S/43S complex assembly in vivo: Overall, initiation was not affected, as assessed by polysome/monosome ratio, and the association of eIF2 and eIF3 with 40S subunits was not affected, as determined from cross-linking analyses. Cheung et al. (2007) reconcile the appearance of a $\mathrm{Gcd}^{-}$phenotype, despite the absence of evidence for overall effects of this mutation on initiation, with the idea that the effect of 9,12 on TC loading is modest, and that translation initiation at the GCN4 start codon is extremely sensitive to TC loading. This is because in the absence of loaded TC, ribosomes that resume scanning after translation of uORF1 will skip subsequent uORF start codons.

The differences between the in vitro and in vivo data suggest the possibility that assembly of the PIC is more robust in vivo. This may be explained in part by additional interactions of eIF1 with eIF3, which is not present in the in vitro experiments. Consistent with this possibility, Valásek et al. (2004) showed previously that the NTT of an eIF3 subunit (eIF3c) contributes to eIF1 recruitment to the ribosome and to ribosomal function in vivo (see below). Other mutations within the eIF1NTT caused a Gcd ${ }^{-}$phenotype that is not suppressed by overexpression of $\mathrm{TC}$, and in experiments at a higher growth temperature, some of them impaired the ablility of the ribosome to scan after uORF1 translation (M. Reibarkh, Y. Yamamoto, C.R. Singh, F. del Rio, B. Lee, R.E. Luna, M. Ii, G. Wagner, and K. Asano, in prep.), indicating that we do not yet have a complete explanation for how subtle eIF1 effects on PIC assembly in vivo lead to a $\mathrm{Gcd}^{-}$phenotype. For example, these data suggest a new idea that certain $\mathrm{Gcd}^{-}$phenotypes could arise from faulty recognition of UORF AUG codons so that even when TC is loaded after translation of uORF1, the scanning ribosome would skip subsequent uORF start codons.

\section{Tales of tails: roles of unstructured terminal tails in eukaryotic initiation}

A variety of studies are helping to provide a picture of how initiation factors bind to the $40 \mathrm{~S}$ subunit. Cryo-EM analysis (Siridechadilok et al. 2005) has been used to locate human eIF3 and eIF4G (the major adaptor subunit of eIF4F) on the 40S subunit (Fig. 2). The location of eIF1 is predicted by analyses of data from hydroxyl-radical mapping studies that positioned human eIF1 with respect to the $40 \mathrm{~S}$ ribosome subunit and tRNA ${ }_{\mathrm{i}}{ }^{\text {Met }}$ (Lomakin et al. 2003). In addition, the above-mentioned structural studies by the Asano and Wagner laboratories (M. Reibarkh, Y. Yamamoto, C.R. Singh, F. del Rio, B. Lee, R.E. Luna, M. Ii, G. Wagner, and K. Asano, in prep.) place eIF5-CTD in a narrow space surrounded by eIF1, $\operatorname{tRNA}_{i}{ }^{\mathrm{Met}}$, and the

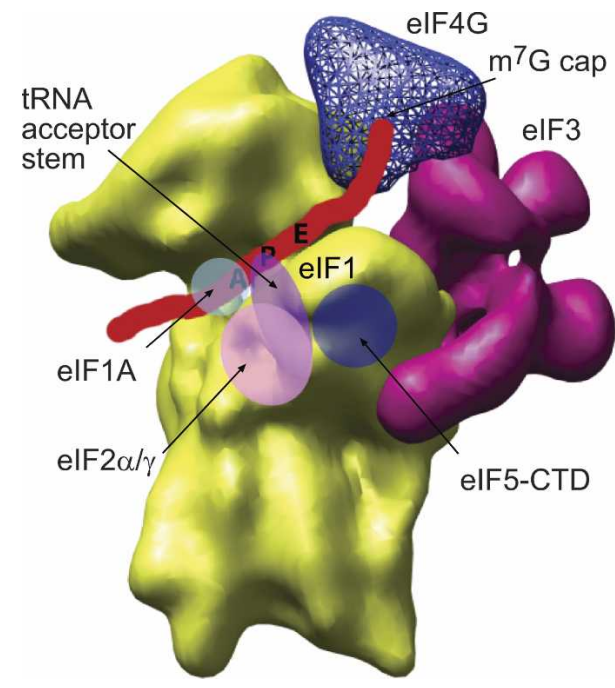

Figure 2. Predicted location of eIFs on the $40 \mathrm{~S}$ subunit. The cryo-EM image of 40S/eIF3/eIF4G complex with mRNA is shown in red and the eIF1-binding face labeled as eIF1 (from Siridechadilok et al. 2005; reprinted with permission from AAS) is overlaid with differently colored circles representing approximate locations of eIF1A, eIF $2 \alpha / \gamma$, eIF5-CTD, and the acceptor stem of tRNA from available information (see the text). Note that the location of $\mathrm{tRNA}_{\mathrm{i}}{ }^{\mathrm{Met}}$ is purely based on its anti-codon binding to the 40S P site. It is not clear whether tRNA ${ }_{i}{ }^{\text {Met }}$ at this stage is bound to the $\mathrm{P}$ site in a manner similar to the P-site tRNA in elongation, or is in the $\mathrm{P} / \mathrm{I}$ configuration as found in the bacterial initiation complex (Allen and Frank 2007). Thus, the location of eIF $2 \alpha / \gamma$ is more approximate than that of eIF5-CTD, which is shown to directly bind eIF1 at the basic and hydrophobic surface altered by mutations M4 and 93-97, respectively (see the text). Yatime et al. (2006) predicted that archaeal aIF $2 \alpha$ contacts helix 44 near the $\mathrm{P}$ site as shown, consistent with a recent report on cross-linking of the mRNA -3 position to eIF2 $\alpha$ (Pisarev et al. 2006).

40S subunit, as shown by the dark-purple circle in Figure 2. As for the location of eIF2, it has been established that eIF2 $\gamma$ displays a high sequence similarity to bacterial elongation factor 1A (EF-Tu) (for review, see Hinnebusch et al. 2007), and the crystal structure of the latter in complex with GTP and aminoacyl tRNA is known (Nissen et al. 1995). Based on these data, Yatime et al. (2006) presented a docking model of archaeal aIF2 $\gamma / \alpha$ complex with tRNA based on their solution of the crystal structure of the two polypeptides, and the structure of bacterial elongation factor $1 \mathrm{~A}$ (EF-Tu) with tRNA. The structure obtained is consistent with the results of genetic and biochemical studies on eIF2 $\gamma$ in yeast (Hinnebusch et al. 2007). The eIF5B-binding site has been inferred by comparison with the location of bacterial IF2 (which is homologous in structure and function to eIF5B) in the PIC (Allen and Frank 2007). Very recently, cryo-EM mapping was used to place yeast eIF1 and eIF1A on the $40 \mathrm{~S}$ subunit, with eIF1A likely occupying the A site (Passmore et al. 2007). Together, these studies provide sufficient information to deduce the location of major domains of tRNA ${ }_{i}{ }^{\text {Met }}$, eIF1, eIF1A, eIF2, eIF3, eIF4G, eIF5, and eIF5B on the $40 \mathrm{~S}$ subunit (Fig. 2). 
The combined picture indicates that the factors cover a limited space of the $40 \mathrm{~S}$ subunit surface, with eIF1, eIF1A, eIF2, and eIF5 at or near the decoding site. This is consistent with the direct use of these four factors in AUG recognition by the initiating ribosome. While these models need to be substantiated with real structures, the combined picture thus far presents the important question of how the individual eIFs communicate with each other on the ribosome. eIFs characteristically have unstructured tails; some of these, such as the eIF1-NTT, are clearly important for initiation. Understanding the physical interactions that occur through these unstructured tails therefore will also be important for understanding how eIFs communicate with each other and the ribosome.

The best characterized unstructured tails are found in eIF1A. eIF1A consists of a $\beta$-barrel structure similar to bacterial IF1, a C-terminally adjacent helical element that packs against the $\beta$ barrel, an unstructured NTT, and an unstructured C-terminal tail (CTT) (Battiste et al. 2000; Marintchev et al. 2003; Olsen et al. 2003). eIF1ANTT directly binds eIF2 and eIF3, major MFC components, to promote initial 43S complex assembly (Olsen et al. 2003). eIF1A-CTT directly binds eIF5B (Marintchev et al. 2003), and a recent report strongly indicates that residues 149-153 of yeast eIF1A-CTT facilitate the binding of eIF5B to the $40 \mathrm{~S}$ subunit. This occurs after the steps of AUG selection and the release of (at least) eIF1, $P_{i}$, and eIF2-GDP (Fringer et al. 2007). Mutational analyses of residues in the eIF1A-CTT show that F131 and F133 can also be important for promoting TC loading on the $43 \mathrm{~S}^{\star}$ and $48 \mathrm{~S}^{\star}$ in vitro in a manner dependent on eIF1 bound to the 40S subunit (Fekete et al. 2007). Thus, eIF1A-CTT also appears to promote $43 \mathrm{~S}$ complex assembly after the $40 \mathrm{~S}$ subunit binds eIF1. eIF1A $(131,133)$ confers a Sui ${ }^{-}$phenotype in vivo, as does deletion of eIF1ACTT (Fekete et al. 2005), further suggesting that F131 and F133 directly regulate the 40S subunit and likely suppress the ribosome conformational change to a closed form. Hence, mutating the eIF1A-CTT enhances the closed conformation at non-AUGs.

As noted above, the eIF1A-NTT mutation 17-21 has an opposite effect on AUG selection, suppressing Suiphenotypes caused by eIF5 and eIF $2 \beta$ mutations and decreasing leaky scanning past AUG codons (Fekete et al. 2007). This indicates that the wild-type eIF1A-NTT could promote the change to the closed conformation at either AUG or UUG codons.

In the model proposed by Fekete et al. (2007), both the tails of eIF1A are postulated to play critical roles in regulating ribosome conformational changes: The interaction of eIF1A-CTT with the ribosome favors the open, scanning-competent conformation, whereas the interaction of eIF1A-NTT favors the closed conformation that subsequently triggers factor release. This conformational change could involve switching of the eIF1A-CTT partner from the ribosome to eIF5 (Maag et al. 2006). The interactions of the 40S subunit with eIF1A tails as well as that with eIF1-NTT, as proposed above, are hypothetical, and their demonstration would be the attractive ma- jor target of further study. It will also be interesting to determine whether the postulated interactions of eIF1NTT, eIF1A-NTT, and eIF1A-CTT with the ribosome and with components of the MFC occur simultaneously, or whether such interactions are mutually exclusive.

Altogether, recent studies on eIF1 and eIF1A strongly suggest that the interactions of their unstructured tails play important roles in multiple steps of translation initiation: (1) MFC-mediated initial 43S/48S complex assembly (eIF1-NTT, eIF1A-NTT); (2) formation of a scanning-competent complex (eIF1A-CTT); (3) transition to a closed conformation in response to AUG selection (eIF1A-NTT and possibly eIF1-NTT); and (4) 60S subunit joining (eIF1A-CTT binding to eIF5B). The proposal that eIF5B binds to the ribosome after eIF2 is released (Fringer et al. 2007), and therefore subsequent to AUG selection, is consistent with eIF1A-CTT functioning at different stages of initiation to recruit eIF2 and eIF5B. Differential timing of eIF2 and eIF5B binding also resolves a major steric clash that would be predicted to arise from simultaneous binding of eIF2 and eIF5B to the CCA end of the tRNA, based on current understanding of where these factors and tRNA associate with the ribosome (see Fig. 2; Allen and Frank 2007).

\section{Translational control by MFC}

In light of the present study (Cheung et al. 2007), it is possible to deduce the effect of eIF3 on the presumed conformational changes associated with the switch from an open to closed conformation. The serine-rich acidic NTT (residues 1-156) of eIF3c binds tightly to eIF1. Mutations altering different segments of the eIF3c-NTT confer a $\mathrm{Gcd}^{-}$phenotype in a gcn2s strain and also can confer a Sui ${ }^{-}$phenotype; the latter phenotype can be suppressed by overexpression of eIF1 (Valásek et al. 2004). These results indicate that the eIF3c-NTT/eIF1 interaction persists throughout the initiation process, mediating both the initial MFC assembly and accurate start codon selection. Interestingly, two mutations altering the serine-rich or acidic segments of eIF3c-NTT suppress the Sui- phenotype of sui1-1-D83G (Valásek et al. 2004). Thus, interaction of eIF3c-NTT with components of the PIC may influence the conformational change to a closed conformation.

A regulatory role is also postulated for eIF $2 \beta-\mathrm{NTT}$, as its binding to the eIF5-CTD greatly increases the latter's affinity for eIF3c-NTT; these interactions are important to promote initial MFC assembly (Singh et al. 2004). A variety of studies show that rapid MFC assembly is facilitated by mutually cooperative interactions involving eIF1, eIF5-CTD, and NTTs of eIF3c and eIF2 $\beta$ (for review, see Hinnebusch et al. 2007). Furthermore, eIF2 and eIF3 bind directly together to promote MFC formation (Valásek et al. 2002), even though their binding sites on the $40 \mathrm{~S}$ subunit appear to be separated (Fig. 2). Finally, there is indirect evidence that the initiator tRNA is an important element promoting MFC assembly (Singh et al. 2006). Taken together, these results suggest an attractive idea that mutual cooperative interactions between 
the MFC constituents promote formation of MFC in a packed conformation. In this regard, recent reports from the Asano, Pavitt, and Anderson laboratories present evidence that interaction between eIF2 $\beta$-NTT and eIF5CTD mediates formation of a second complex composed of eIF2, eIF5, and perhaps GDP (Singh et al. 2007) and that this complex plays a role in antagonizing guanine nucleotide exchange catalyzed by eIF2B (Singh et al. 2006). While the regulatory role played by the eIF2/eIF5 complex remains to be established, this finding raises an interesting possibility that the packed configuration of the MFC also facilitates discrimination against the interaction of MFC partners with eIF2 •GDP.

\section{Concluding remarks}

The findings of Cheung et al. (2007) in the previous issue provide new insights into the assembly, structure, and function of the ribosomal PIC and the role of eIF1 at different steps in translation initiation. The complementary use of biochemical and biophysical assays with genetic and phenotypic assays, coupled with knowledge of structure, enables a greater understanding of how the scanning ribosome selects an appropriate start codon. Now it is nearly 30 years since the original elaboration of the scanning model, and a mechanistic understanding of how a start codon is selected by the scanning ribosome is coming into view.

\section{Acknowledgments}

We thank Mikhail Reibarkh and Gerhard Wagner for communicating unpublished results and Chris Fraser and Jennifer Doudna for providing the image used in Figure 2. We regret that, due to constraints, there are relevant communications that have not been cited here. K.A. and M.S.S. are supported by grants from the National Institutes of Health.

\section{References}

Algire, M.A., Maag, D., and Lorsch, J.R. 2005. P $_{\mathrm{i}}$ release from eIF2, not GTP hydrolysis, is the step controlled by start-site selection during eukaryotic translation initiation. Mol. Cell 20: 251-262.

Allen, G.S. and Frank, J. 2007. Structural insights on the translation initiation complex: Ghosts of a universal initiation complex. Mol. Microbiol. 63: 941-950.

Asano, K., Clayton, J., Shalev, A., and Hinnebusch, A.G. 2000. A multifactor complex of eukaryotic initiation factors, eIF1, eIF2, eIF3, eIF5, and initiator tRNA(Met) is an important translation initiation intermediate in vivo. Genes \& Dev. 14: 2534-2546.

Battiste, J.L., Pestova, T.V., Hellen, C.U., and Wagner, G. 2000. The eIF1A solution structure reveals a large RNA-binding surface important for scanning function. Mol. Cell 5: 109119.

Castilho-Valavicius, B., Yoon, H., and Donahue, T.F. 1990. Genetic characterization of the Saccharomyces cerevisiae translational initiation suppressors sui1, sui2 and SUI3 and their effects on HIS4 expression. Genetics 124: 483-495.

Cheung, Y.-N., Maag, D., Mitchell, S.F., Fekete, C.A., Algire,
M.A., Takacs, J.E., Shirokikh, N., Pestova, T., Lorsch, J.R., and Hinnebusch, A.G. 2007. Dissociation of eIF1 from the 40S ribosomal subunit is a key step in start codon selection in vivo. Genes \& Dev. 21: 1217-1230.

Das, S., Ghosh, R., and Maitra, U. 2001. Eukaryotic translation initiation factor 5 functions as a GTPase-activating protein. J. Biol. Chem. 276: 6720-6726.

Donahue, T.F. 2000. Genetic approaches to translation initiation in Saccharomyces cerevisiae. In Translational control of gene expression (eds. N. Sonenberg et al.), pp. 595-614. Cold Spring Harbor Laboratory Press, Cold Spring Harbor, NY.

Donahue, T.F. and Cigan, A.M. 1988. Genetic selection for mutations that reduce or abolish ribosomal recognition of the HIS4 translational initiator region. Mol. Cell. Biol. 8: 29552963.

Donahue, T.F., Cigan, A.M., Pabich, E.K., and Valavicius, B.C. 1988. Mutations at a $\mathrm{Zn}$ (II) finger motif in the yeast eIF-2 $\beta$ gene alter ribosomal start-site selection during the scanning process. Cell 54: 621-632.

Fekete, C.A., Applefield, D.J., Blakely, S.A., Shirokikh, N., Pestova, T., Lorsch, J.R., and Hinnebusch, A.G. 2005. The eIF1A C-terminal domain promotes initiation complex assembly, scanning and AUG selection in vivo. EMBO I. 24: 3588-3601.

Fekete, C.A., Mitchell, S.F., Cherkasova, V.A., Applefield, D., Algire, M.A., Maag, D., Saini, A.K., Lorsch, J.R., and Hinnebusch, A.G. 2007. N- and C-terminal residues of eIF1A have opposing effects on the fidelity of start codon selection. EMBO I. 26: 1602-1614.

Fletcher, C.M., Pestova, T.V., Hellen, C.U., and Wagner, G. 1999. Structure and interactions of the translation initiation factor eIF1. EMBO I. 18: 2631-2637.

Fringer, J.M., Acker, M.G., Fekete, C.A., Lorsch, J.R., and Dever, T.E. 2007. Coupled release of eukaryotic translation initiation factors $5 \mathrm{~B}$ and $1 \mathrm{~A}$ from $80 \mathrm{~S}$ ribosomes following subunit joining. Mol. Cell. Biol. 27: 2384-2397.

He, H., von der Haar, T., Singh, C.R., Ii, M., Li, B., Hinnebusch, A.G., McCarthy, J.E., and Asano, K. 2003. The yeast eukaryotic initiation factor 4G (eIF4G) HEAT domain interacts with eIF1 and eIF5 and is involved in stringent AUG selection. Mol. Cell. Biol. 23: 5431-5445.

Hinnebusch, A.G. 2005. Translational regulation of GCN4 and the general amino acid control of yeast. Annu. Rev. Microbiol. 59: 407-450.

Hinnebusch, A.G. 2006. eIF3: A versatile scaffold for translation initiation complexes. Trends Biochem. Sci. 31: 553-562.

Hinnebusch, A.G., Dever, T.E., and Asano, K. 2007. Mechanism of translation initiation in the yeast Saccharomyces cerevisiae. In Translational control in biology and medicine (eds. M.B. Mathews et al.), pp. 225-268. Cold Spring Harbor Laboratory Press, Cold Spring Harbor, NY.

Huang, H.K., Yoon, H., Hannig, E.M., and Donahue, T.F. 1997. GTP hydrolysis controls stringent selection of the AUG start codon during translation initiation in Saccharomyces cerevisiae. Genes \& Dev. 11: 2396-2413.

Jivotovskaya, A.V., Valasek, L., Hinnebusch, A.G., and Nielsen, K.H. 2006. Eukaryotic translation initiation factor 3 (eIF3) and eIF2 can promote mRNA binding to 40S subunits independently of eIF4G in yeast. Mol. Cell. Biol. 26: 1355-1372.

Kapp, L.D. and Lorsch, J.R. 2004. The molecular mechanics of eukaryotic translation. Annu. Rev. Biochem. 73: 657-704.

Kolupaeva, V.G., Unbehaun, A., Lomakin, I.B., Hellen, C.U., and Pestova, T.V. 2005. Binding of eukaryotic initiation factor 3 to ribosomal 40 S subunits and its role in ribosomal dissociation and anti-association. RNA 11: 470-486. 
Kozak, M. 1978. How do eucaryotic ribosomes select initiation regions in messenger RNA? Cell 15: 1109-1123.

LeFebvre, A.K., Korneeva, N.L., Trutschl, M., Cvek, U., Duzan, R.D., Bradley, C.A., Hershey, J.W., and Rhoads, R.E. 2006. Translation initiation factor eIF4G-1 binds to eIF3 through the eIF3e subunit. J. Biol. Chem. 281: 22917-22932.

Lomakin, I.B., Kolupaeva, V.G., Marintchev, A., Wagner, G., and Pestova, T.V. 2003. Position of eukaryotic initiation factor eIF1 on the 40S ribosomal subunit determined by directed hydroxyl radical probing. Genes \& Dev. 17: 27862797.

Maag, D., Fekete, C.A., Gryczynski, Z., and Lorsch, J.R. 2005. A conformational change in the eukaryotic translation preinitiation complex and release of eIF1 signal recognition of the start codon. Mol. Cell 17: 265-275.

Maag, D., Algire, M.A., and Lorsch, J.R. 2006. Communication between eukaryotic translation initiation factors 5 and $1 \mathrm{~A}$ within the ribosomal pre-initiation complex plays a role in start site selection. J. Mol. Biol. 356: 724-737.

Majumdar, R. and Maitra, U. 2005. Regulation of GTP hydrolysis prior to ribosomal AUG selection during eukaryotic translation initiation. EMBO J. 24: 3737-3746.

Marintchev, A., Kolupaeva, V.G., Pestova, T.V., and Wagner, G. 2003. Mapping the binding interface between human eukaryotic initiation factors $1 \mathrm{~A}$ and $5 \mathrm{~B}$ : A new interaction between old partners. Proc. Natl. Acad. Sci. 100: 1535-1540.

Nissen, P., Kjeldgaard, M., Thirup, S., Polekhina, G., Reshetnikova, L., Clark, B.F., and Nyborg, J. 1995. Crystal structure of the ternary complex of Phe-tRNA ${ }^{\text {Phe }}$, EF-Tu, and a GTP analog. Science 270: 1464-1472.

Olsen, D.S., Savner, E.M., Mathew, A., Zhang, F., Krishnamoorthy, T., Phan, L., and Hinnebusch, A.G. 2003. Domains of eIF1A that mediate binding to eIF2, eIF3 and eIF5B and promote ternary complex recruitment in vivo. EMBO J. 22: 193204.

Passmore, L.A., Schmeing, T.M., Maag, D., Applefield, D.J., Acker, M.G., Algire, M.A., Lorsch, J.R., and Ramakrishnan, V. 2007. The eukaryotic translation initiation factors eIF1 and eIF1A induce an open conformation of the $40 \mathrm{~S}$ ribosome. Mol. Cell 26: 41-50.

Paulin, F.E., Campbell, L.E., O’Brien, K., Loughlin, J., and Proud, C.G. 2001. Eukaryotic translation initiation factor 5 (eIF5) acts as a classical GTPase-activator protein. Curr. Biol. 11: 55-59.

Pestova, T.V. and Kolupaeva, V.G. 2002. The roles of individual eukaryotic translation initiation factors in ribosomal scanning and initiation codon selection. Genes \& Dev. 16: 29062922.

Pestova, T.V., Borukhov, S.I., and Hellen, C.U. 1998. Eukaryotic ribosomes require initiation factors 1 and $1 \mathrm{~A}$ to locate initiation codons. Nature 394: 854-859.

Pestova, T.V., Lorsch, J.R., and Hellen, C.U.T. 2007. The mechanism of translation initiation in eukaryotes. In Translational control in biology and medicine (eds. M.B. Mathews et al.), pp. 87-128. Cold Spring Harbor Laboratory Press, Cold Spring Harbor, NY.

Pisarev, A.V., Kolupaeva, V.G., Pisareva, V.P., Merrick, W.C., Hellen, C.U., and Pestova, T.V. 2006. Specific functional interactions of nucleotides at key -3 and +4 positions flanking the initiation codon with components of the mammalian 48S translation initiation complex. Genes \& Dev. 20: 624636.

Singh, C.R., He, H., Ii, M., Yamamoto, Y., and Asano, K. 2004. Efficient incorporation of eukaryotic initiation factor 1 into the multifactor complex is critical for formation of functional ribosomal preinitiation complexes in vivo. I. Biol.
Chem. 279: 31910-31920.

Singh, C.R., Lee, B., Udagawa, T., Mohammad-Qureshi, S.S., Yamamoto, Y., Pavitt, G.D., and Asano, K. 2006. An eIF5/ eIF2 complex antagonizes guanine nucleotide exchange by eIF2B during translation initiation. EMBO J. 25: 4537-4546.

Singh, C.R., Udagawa, T., Lee, B., Wassink, S., He, H., Yamamoto, Y., Anderson, J.T., Pavitt, G.D., and Asano, K. 2007. Change in nutritional status modulates the abundance of critical pre-initiation intermediate complexes during translation initiation in vivo. J. Mol. Biol. (in press).

Siridechadilok, B., Fraser, C.S., Hall, R.J., Doudna, J.A., and Nogales, E. 2005. Structural roles for human translation factor eIF3 in initiation of protein synthesis. Science 310: 15131515.

Stewart, J.W., Sherman, F., Shipman, N.A., and Jackson, M. 1971. Identification and mutational relocation of the AUG codon initiating translation of iso-1-cytochrome $c$ in yeast. $J$. Biol. Chem. 246: 7429-7445.

Unbehaun, A., Borukhov, S.I., Hellen, C.U., and Pestova, T.V. 2004. Release of initiation factors from $48 \mathrm{~S}$ complexes during ribosomal subunit joining and the link between establishment of codon-anticodon base-pairing and hydrolysis of eIF2-bound GTP. Genes \& Dev. 18: 3078-3093.

Valásek, L., Nielsen, K.H., and Hinnebusch, A.G. 2002. Direct eIF2-eIF3 contact in the multifactor complex is important for translation initiation in vivo. EMBO J. 21: 5886-5898.

Valásek, L., Nielsen, K.H., Zhang, F., Fekete, C.A., and Hinnebusch, A.G. 2004. Interactions of eukaryotic translation initiation factor 3 (eIF3) subunit NIP1/c with eIF1 and eIF5 promote preinitiation complex assembly and regulate start codon selection. Mol. Cell. Biol. 24: 9437-9455.

Yatime, L., Mechulam, Y., Blanquet, S., and Schmitt, E. 2006. Structural switch of the $\gamma$ subunit in an archaeal aIF2 $\alpha \gamma$ heterodimer. Structure 14: 119-128.

Yoon, H.J. and Donahue, T.F. 1992. The suil suppressor locus in Saccharomyces cerevisiae encodes a translation factor that functions during $\mathrm{tRNA}_{\mathrm{i}}{ }^{\mathrm{Met}}$ recognition of the start codon. Mol. Cell Biol. 12: 248-260. 


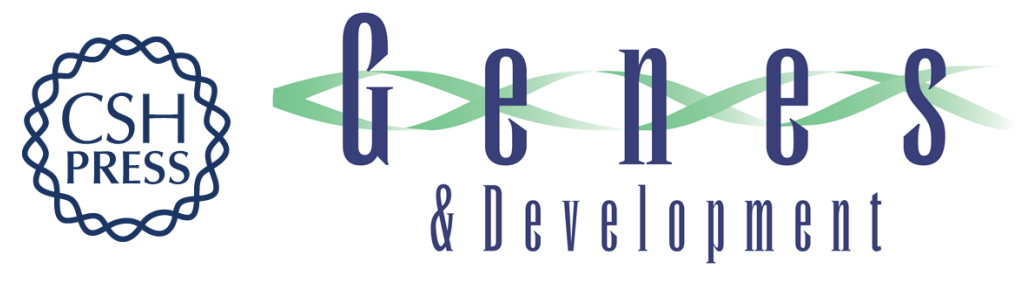

\section{Translation factor control of ribosome conformation during start codon selection}

Katsura Asano and Matthew S. Sachs

Genes Dev. 2007, 21:

Access the most recent version at doi:10.1101/gad.1562707
Related Content Dissociation of elF1 from the 40S ribosomal subunit is a key step in start codon selection in vivo
Yuen-Nei Cheung, David Maag, Sarah F. Mitchell, et al.
Genes Dev. May, 2007 21: 1217-1230
References This article cites 43 articles, 27 of which can be accessed free at:
http://genesdev.cshlp.org/content/21/11/1280.full.html\#ref-list-1
Articles cited in:
http://genesdev.cshlp.org/content/21/11/1280.full.html\#related-urls

\section{License}
Email Alerting
Service
Receive free email alerts when new articles cite this article - sign up in the box at the top right corner of the article or click here.

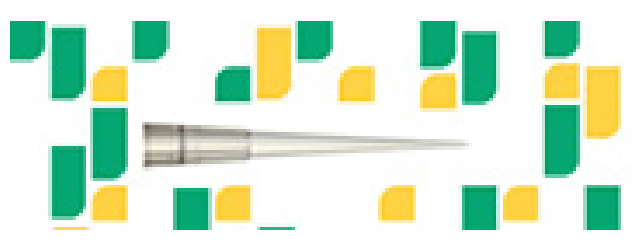

Focused on your science. 